\title{
Spectrotemporal control of soft $x$-ray laser pulses
}

\author{
Najmeh S. Mirian $\odot,{ }^{1}$ Enrico Allaria $\odot,{ }^{1}$ Niky Bruchon $\odot,{ }^{2}$ Paolo Cinquegrana, ${ }^{1}$ \\ Miltcho Boyanov Danailov, ${ }^{1}$ Giovanni De Ninno, ${ }^{1,3}$ Simone Di Mitri®, ${ }^{1}$ Eugenio Ferrari, ${ }^{4}$ \\ Luca Giannessi® ${ }^{1,5}$ Erik Hemsing $\odot,{ }^{6}$ Giuseppe Penco, ${ }^{1}$ Eléonore Roussel $\odot,{ }^{7}$ Simone Spampinati, ${ }^{1}$ \\ Carlo Spezzani, ${ }^{1}$ Mauro Trovó, ${ }^{1}$ Marco Veronese, ${ }^{1}$ and Primož Rebernik Ribič $\oplus^{1,3,}$ \\ ${ }^{1}$ Elettra-Sincrotrone Trieste, Area Science Park, 34149 Basovizza, Trieste, Italy \\ ${ }^{2}$ Department of Engineering and Architecture, Università degli Studi di Trieste, 34127 Trieste, Italy \\ ${ }^{3}$ Laboratory of Quantum Optics, University of Nova Gorica, 5000 Nova Gorica, Slovenia \\ ${ }^{4}$ Paul Scherrer Institut, 5232 Villigen PSI, Switzerland \\ ${ }^{5}$ Istituto Nazionale di Fisica Nucleare - Laboratori Nazionali di Frascati, 00044 Frascati, Rome, Italy \\ ${ }^{6}$ SLAC National Accelerator Laboratory, Menlo Park, California 94025, USA \\ ${ }^{7}$ Université Lille, CNRS, UMR 8523 - PhLAM - Physique des Lasers Atomes et Molécules, \\ F-59000 Lille, France
}

(Received 9 January 2020; accepted 20 May 2020; published 11 June 2020)

\begin{abstract}
We exploit echo-enabled harmonic generation (EEHG) to produce fully coherent free-electron laser (FEL) pulses at soft-x-ray wavelengths and shape their spectrotemporal content. In an EEHG FEL, the longitudinal phase space of the relativistic electron beam that amplifies light is precisely tailored using two external seed lasers and two magnetic chicanes. We show that the spectrotemporal properties of the emitted radiation can be controlled by tuning the bandwidth, linear frequency chirp, and intensity of one of the seed lasers. The experimental data are supported by analytical and numerical models. Our results open a pathway toward coherent control of quantum processes at short wavelengths in the fields of applied physics, chemistry and biology, where manipulating the radiation spectrum is essential. The ability to precisely control the spectrotemporal content of intense, short-wavelength FEL pulses and the low sensitivity of the radiation to electron-beam imperfections make the technique an ideal candidate for use in chirped-pulse amplification schemes.
\end{abstract}

DOI: 10.1103/PhysRevAccelBeams.23.060701

\section{INTRODUCTION}

The development of pulsed lasers and pulse shaping techniques has equipped researchers with tools to probe and control quantum dynamical processes, giving birth to a new research field called quantum coherent control [1-3]. Advances in generation and manipulation of short laser pulses have led to a rapid progress in the field, making it possible to investigate and manipulate quantum interference phenomena with a wide range of applications in chemical reactions, quantum information processing, spectroscopy, and ultracold physics [4-7].

With the advent of high-gain free-electron lasers (FELs) $[8,9]$, short and powerful pulses in the $\mathrm{x}$-ray spectral range became available, making it possible to investigate core electrons in atoms, molecules, and materials, which are not

\footnotetext{
*primoz.rebernik@elettra.eu
}

Published by the American Physical Society under the terms of the Creative Commons Attribution 4.0 International license. Further distribution of this work must maintain attribution to the author(s) and the published article's title, journal citation, and DOI. accessible with conventional lasers. While ultrafast pulse shaping techniques are well established in the visible and infrared (IR), direct application of such methods to an x-ray FEL is not straightforward. The first step toward spectrotemporal control in an FEL is the generation of fully coherent FEL pulses. Most x-ray FELs rely on selfamplified spontaneous emission (SASE) [10], which only guarantees generation of partially coherent pulses, typically characterized by a burst of coherent radiation spikes with a random mutual phase relationship. Generation of fully coherent FEL light can be achieved by triggering the emission with an external laser. Such seeded FELs use a combination of a visible or IR laser, a short magnetic undulator (modulator), and a magnetic chicane to produce nanometer-sized bunching of electrons in a relativistic electron beam, which then emits coherent light at harmonics of the seed laser frequency in the downstream undulator (radiator).

The development of coherent control schemes for seeded FELs in the extreme-ultraviolet (EUV) and x-ray spectral regions has recently started at FERMI [11,12], an externally seeded FEL based on high-gain harmonic generation (HGHG) [13,14]. While HGHG allows generating almost 
fully coherent pulses down to around $20 \mathrm{~nm}$, the sensitivity of the method to electron-beam imperfections in the form of unwanted energy and/or density modulations that may build up during electron-beam acceleration, see, e.g., Ref. [15], degrades the FEL performance in terms of coherence and output stability at shorter wavelengths. To provide a fully coherent and stable output of the FERMI FEL down to $5 \mathrm{~nm}$, we recently implemented a new seeding scheme called echo-enabled harmonic generation (EEHG) $[16,17]$. EEHG is less sensitive to the initial electron-beam properties and should allow generation of stable and coherent light at much higher harmonics of the seed [16-21], making it possible to extend the methods of coherent control to x-rays.

Recent experiments at FERMI have demonstrated amplification of FEL pulses using EEHG at wavelengths as short as $5 \mathrm{~nm}$ and coherent emission down to $2.6 \mathrm{~nm}$, i.e., the 101st harmonic of a seed operating at $264 \mathrm{~nm}$ [22]. Here, we exploit the EEHG setup at FERMI to investigate the spectrotemporal control capabilities of FEL pulses at high harmonics (36 and 37, output wavelength $\sim 7 \mathrm{~nm}$ ) of the seed laser. We demonstrate that spectral and temporal control over the FEL pulse can be achieved by tuning the seed laser parameters. Such pulses may be used for probing the evolution and dephasing of inner shell electronic coherences using advanced spectroscopic techniques [23].

\section{EXPERIMENTAL SETUP AND THEORY}

The EEHG setup (Fig. 1) is based on two modulating sections, each consisting of a seed laser, short undulator (modulator) to facilitate the interaction of the seed with the electron beam, and a dispersive section. The first seed laser imprints a sinusoidal energy modulation with a wave number $k_{1}$ onto the electron beam in the first modulator (M1). The beam coming out of the strong magnetic chicane (DS1) is longitudinally stretched, resulting in a striated phase space with several energy bands. A second seed is then used to once again periodically modulate the electronbeam energy with a wave number $k_{2}$. After passing through a weaker chicane (DS2), the periodic energy modulation is converted into a density modulation (bunching) with frequency components at high harmonics of the seed.

The evolution of the electron-beam energy-longitudinal coordinate phase space $(p, z)$ through the EEHG line is described by the following equations $[16,17,24]$ :

$p_{1}=p+A_{1}(z) \sin \left[k_{1} z+\psi_{1}(z)\right]+\Delta p_{1}(z)$,

$z_{1}=z+B_{1} p_{1} / k_{1}$,

$p_{2}=p_{1}+A_{2}\left(z_{1}\right) \sin \left[k_{2} z_{1}+\psi_{2}\left(z_{1}\right)\right]+\Delta p_{2}\left(z_{1}\right)$,

$z_{2}=z_{1}+B_{2} p_{2} / k_{1}$,

where $A_{1,2}(z)=\Delta E_{1,2}(z) / \sigma_{E}$ are normalized energy modulations along the beam due to the two seed lasers, $B_{1,2}=k_{1} R_{56}^{(1,2)} \sigma_{E} / E$ are normalized dispersions, $E$ is the electron-beam energy and $\sigma_{E}$ the slice energy spread, $\Delta p_{1,2}(z)$ are additional energy distortions that may be introduced along the linear accelerator (linac) and in the modulating sections (and exclude the seed laser modulations), and $\psi_{1,2}(z)$ are longitudinal phase variations of the first and second seed lasers and include chirp as well as laser phase fluctuations.

Starting from Eq. (1) and following Refs. [24] and [22], the bunching factor, i.e., the Fourier transform of the electron-beam density distribution, which determines the EEHG output around harmonic $a_{E}=n+m k_{2} / k_{1}$, where $n$ and $m$ are integers, is written as:

$$
\begin{aligned}
b(k)= & e^{-\frac{1}{2}\left(\xi_{E}+\frac{k-k_{E}}{k_{1}} B\right)^{2}} \int_{-\infty}^{+\infty} J_{m}\left[-\frac{k}{k_{1}} B_{2} A_{2}(z)\right] \\
& \times J_{n}\left[-\left(\xi_{E}+\frac{k-k_{E}}{k_{1}} B\right) A_{1}(z)\right] e^{i\left(\varphi(z)-\left(k-k_{E}\right) z\right)} d z,
\end{aligned}
$$

where $\xi_{E}=n B_{1}+a_{E} B_{2}$ is the EEHG scaling factor, which governs the performance of the bunching process $[19,24]$,

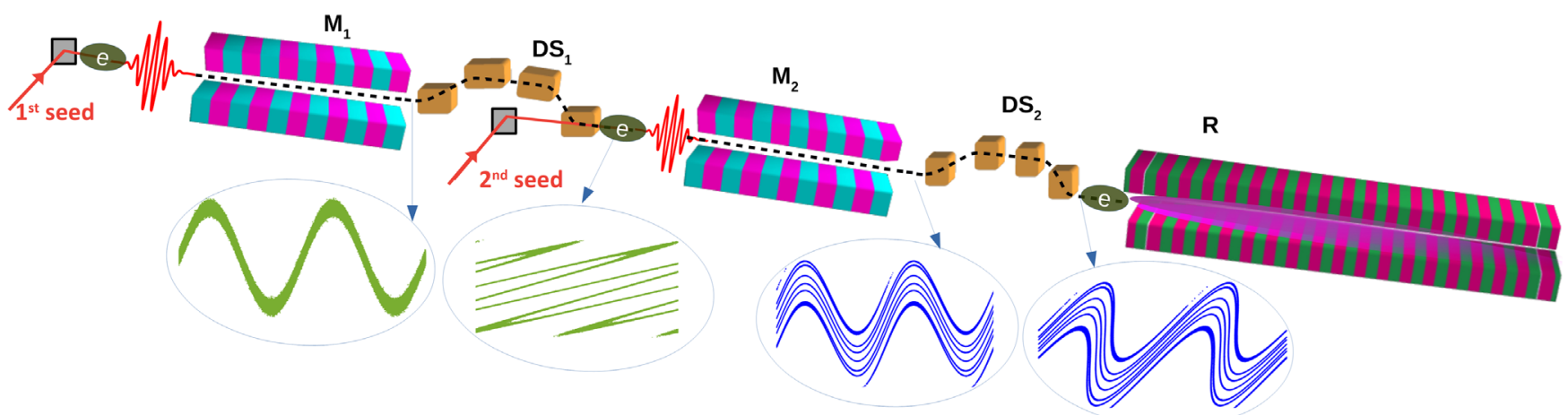

FIG. 1. The components of the EEHG scheme: first modulator (M1), first (strong) dispersive section (DS1), second modulator (M2), and second (weaker) dispersive section (DS2); see text for details. The nanobunched electron beam exiting DS2 is injected into the radiator $(\mathrm{R})$, whose periodic magnetic field forces the electrons to emit coherent and powerful EUV or soft-X-ray pulses. 
$k_{E}=a_{E} k_{1}$ is the bunching (central) wave number, and $B=B_{1}+B_{2}$. The bunching around a specific harmonic $a_{E}$ is optimized by minimizing $\left|\xi_{E}\right|$.

In Eq. (2), the terms that contribute to the bunching phase $\varphi(z)$ are the energy distortions and seed laser phase variations:

$\varphi(z)=-\xi_{E} \Delta p_{1}(z)-a_{E} B_{2} \Delta p_{2}(z)+n \psi_{1}(z)+m \psi_{2}(z)$.

EEHG is typically operated with $m \gg|n|$. This means that the phase variations of the first seed have a small effect on the bunching bandwidth and only decrease the bunching factor [25].

If the first seed pulse is much longer than the second one [24], the bunching bandwidth obtained from the spectrum in Eq. (2) can be evaluated as $\sigma_{k}^{2}=\sigma_{k_{E}}^{2}+\sigma_{\varphi^{\prime}}^{2}$, where $\sigma_{k_{E}}$ is the transform-limited (TL) bandwidth obtained by setting $\varphi(z)=0$, and $\sigma_{\varphi^{\prime}}=\sigma_{d \varphi / d z}$ is the bandwidth due to the nonlinear phase structures. If the main contribution to $\sigma_{\varphi^{\prime}}$ comes from the (second) seed laser linear frequency chirp [26], the relative bandwidth in the case of optimized bunching can be approximated as [24]:

$$
\begin{aligned}
\bar{\sigma}_{\lambda}^{2} & =\left(\sigma_{\lambda} / \lambda_{E}\right)^{2}=\left(\sigma_{k} / k_{E}\right)^{2} \\
& =\frac{4 \bar{\sigma}_{\lambda L}^{2}}{3 x^{2} m^{4 / 3}}\left[1+\frac{3}{4} m^{2 / 3}\left(x^{2}-1\right)\right],
\end{aligned}
$$

where $\bar{\sigma}_{\lambda L}=\sigma_{\lambda L} / \lambda_{L}=\sigma_{k_{2}} / k_{2}$ is the relative seed bandwidth and $x=\tau / \tau_{0} \geq 1$, with $\tau_{0}$ and $\tau$ the TL and chirped seed durations, respectively.

\section{RESULTS AND DISCUSSION}

Figure 2(a) and (b) show a series of single shot spectra from the FERMI FEL operated in the EEHG configuration at harmonic $a_{E}=36$ of a UV seed laser. Prior to emission, the electron beam with a normalized emittance of $1 \mathrm{~mm}$ mrad was accelerated in the linac to an energy of $E=$ $1.3 \mathrm{GeV}(\simeq 270 \mathrm{keV}$ uncorrelated energy spread), compressed to 700 A peak current and prepared using the EEHG scheme shown in Fig. 1. The dispersion of the first chicane $R_{56}^{(1)}$ was $2.25 \mathrm{~mm}$, while that of the second chicane $R_{56}^{(2)}$ was adjusted to optimize bunching at $a_{E}=36$ in the $n=-1$ tune, according to $R_{56}^{(2)} \sim\left(|n| / a_{E}\right) R_{56}^{(1)} \sim 60 \mu \mathrm{m}$. Both seeds were near-TL with full-width half-maximum (FWHM) durations of 120 and $90 \mathrm{fs,}$, respectively for the case in Fig. 2(a) and 175 and 130 fs, respectively for the case in Fig. 2(b), and were operated at $264 \mathrm{~nm}$.

When the second seed duration is 90 fs [Fig. 2(a)], the FEL pulses are expected to be about 30 fs long [27] with an energy of more than $10 \mu \mathrm{J}$ per pulse and root mean square (RMS) intensity fluctuations limited to $20 \%$. The pulse
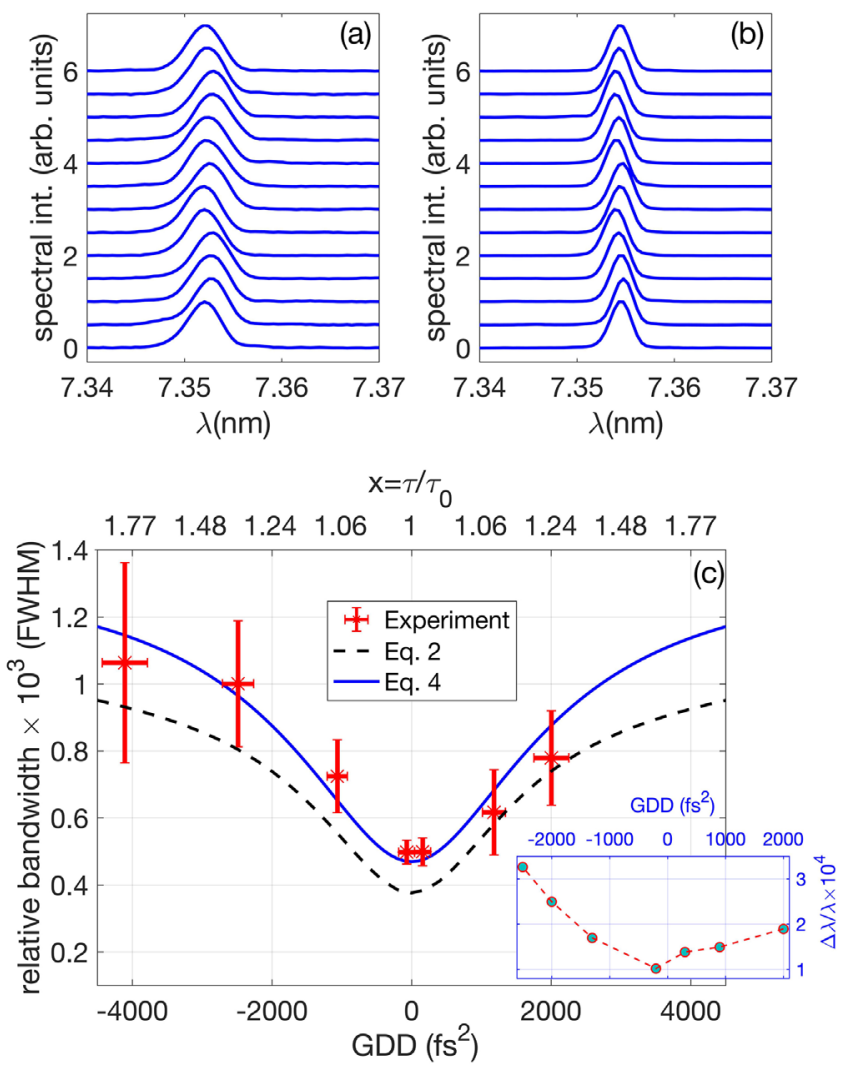

FIG. 2. Normalized single shot EEHG spectra at the 36th harmonic in the $n=-1$ tune for (a) short and (b) long second seed pulses. (c) Relative FEL bandwidth as a function of the second seed GDD: red crosses are experimental data (error bars show RMS fluctuations), while the dashed black and solid blue lines show the relative bandwidths obtained from Eq. (2) and Eq. (4), respectively (see text for details). The inset shows the central wavelength stability as a function of the second seed GDD. The data in c) were acquired in the $n=-2$ tune.

spectra are quasi Gaussian with a relative bandwidth of $5.8 \times 10^{-4}$, about 1.3 the TL-bandwidth obtained by taking the FWHM of the bunching spectral envelope given by Eq. (2), and a high relative central wavelength stability of $4 \times 10^{-5}$.

The first experimental goal was to try decreasing the FEL bandwidth by using a longer [130 fs, Fig. 2(b)] second seed pulse. In this case, the relative bandwidth decreased to $3.4 \times 10^{-4}$, providing a means of controlling the spectral width. As EEHG was shown to be relatively insensitive to electron-beam properties at these wavelengths [22], even longer seed pulses could be utilized, leading to narrower spectra. This is typically not possible when FERMI is operated in the HGHG configuration, where electron-beam imperfections may play a significant role in determining the bandwidth. We note here that for longer seed pulses, the corresponding pulse energies were higher $(\sim 20 \mu \mathrm{J})$ with similar relative intensity fluctuations.

Apart from controlling the FEL bandwidth by adjusting the seed bandwidth (the pulse duration, while keeping the 
pulses near TL), another possibility is to impose a linear frequency chirp on the second seed pulse. We demonstrate this in Fig. 2(c), where we plot the FEL bandwidth as a function of the group delay dispersion (GDD) of the second seed (for the case of $87 \mathrm{fs}$ TL duration), which was controlled by a combination of a fused silica plate and a grating compressor $[28,29]$.

In EEHG, the second seed energy modulation scales as $A_{2} \sim 1 / R_{56}^{(2)}$. To allow optimizing the bunching also for chirped (stretched) seed pulses with a lower peak power (lower $A_{2}$ ), the data in Fig. 2(c) were acquired in the $n=-2$ tune with the second $R_{56}$ increased to $\sim 120 \mu \mathrm{m}$. For zero GDD, the FEL pulses are close to TL-their widths are around 30\% higher than the bandwidth calculated from Eq. (2) by setting $\varphi(z)=0$. Increasing the seed laser GDD to $\sim 3000 \mathrm{fs}^{2}$ allows doubling the FEL bandwidth [30]. The experimental data is reproduced well using the analytical expression in Eq. (4), which assumes that the bunching phase comes only from the second seed laser chirp. This confirms the low sensitivity of EEHG to electron-beam properties. The properties of the FEL spectra are therefore mainly determined by the properties of the seed laser, such as the central wavelength, duration, and chirp. However, Eq. (4) is an approximation [24] and slightly overestimates the bunching bandwidth. A more accurate trend of the TL spectral widths is provided using Eq. (2) and neglecting the energy distortions $\Delta p_{1,2}(z)$. The small discrepancy between the experimental data and the black dashed line in Fig. 2(c) may come from the electronbeam instabilities, which give rise to energy distortions $\Delta p_{1,2}$, seed laser phase instabilities (not taken into account in the calculations), and a nonzero spectrometer resolution. The seed laser phase instabilities become more important for longer seed pulses (higher GDDs). In addition, for longer seed durations the modulation occurs on a larger portion of the electron beam, putting more weight on the energy distortions $\Delta p_{1,2}(z)$. This is confirmed by larger error bars at higher GDDs and by the inset in Fig. 2(c), which shows a decreasing relative central wavelength stability with increasing GDD, although the wavelength stability is still high and is maintained on the $1 \times 10^{-4}$ level.

In the final step, we demonstrate the ability to tune the spectro-temporal content of FEL pulses by adjusting the EEHG parameters, extending the methods developed in Ref. [31] to soft-x-rays. Figure 3 shows the spectral maps (spectrum vs second seed intensity) for different values of the second seed GDD; the insets are theoretical spectral maps obtained from Eq. (2) and agree relatively well with the experiment. In Fig. 3(a), we plot the results for a relatively high (absolute) value of the GDD $=-4100 \mathrm{fs}^{2}$. Starting from the optimum energy modulation that maximizes bunching $\left(A_{2} \approx 1.8\right)$, increasing the seed intensity, the spectrum initially characterized by a single peak broadens until it develops intensity modulations as a function of the wavelength due to the process of electron
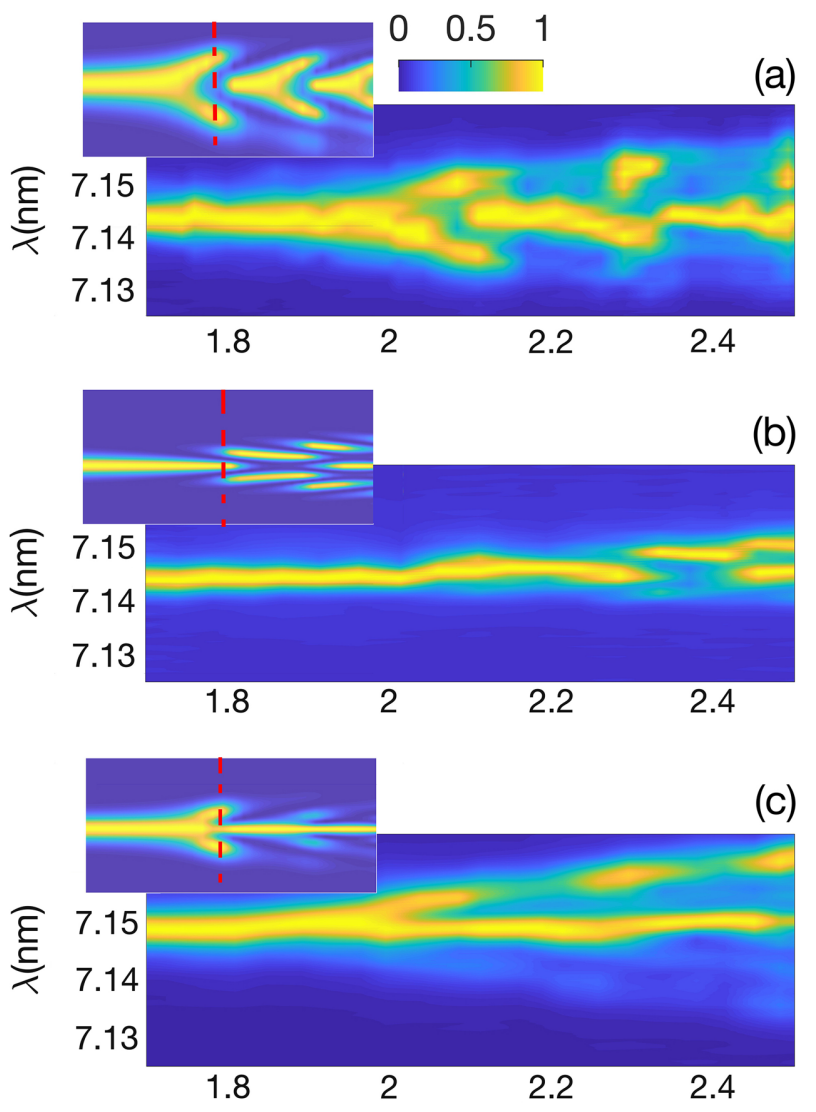

(d)

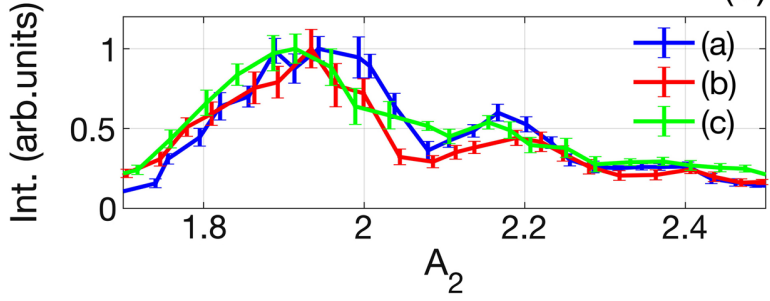

FIG. 3. FEL spectrum vs seed modulation amplitude $A_{2}$ for a GDD of (a) $-4100 \mathrm{fs}^{2}$, (b) $0 \mathrm{fs}^{2}$, and (c) $+2000 \mathrm{fs}^{2}$. The spectral maps were obtained by averaging the FEL spectrum at harmonic 37 over 50 shots and normalizing the result for each value of $A_{2}$. Insets show calculated spectral maps reproduced using Eq. (2). In all cases, $A_{1} \simeq 4, B_{1}=11.4$, while $B_{2}$ was adjusted to maximize bunching. The experimental spectral maps at short wavelengths in (b) and (c) are less intense probably due to the FEL gain (not taken into account in the calculations), as well as due to a possible small mismatch between the optimum and actual EEHG parameters. The relative variation of the FEL intensity with $A_{2}$ for all cases is shown in (d).

overbunching and rebunching [31]. For such high GDD, these intensity modulations directly correspond to the intensity modulations in the temporal domain due to the spectrotemporal equivalence $[32,33]$. A split spectrum in the wavelength domain, indicated by the vertical dashed line in the inset of Fig. 3(a), thus corresponds to a two pulse structure with an overall negative quadratic chirp in the 

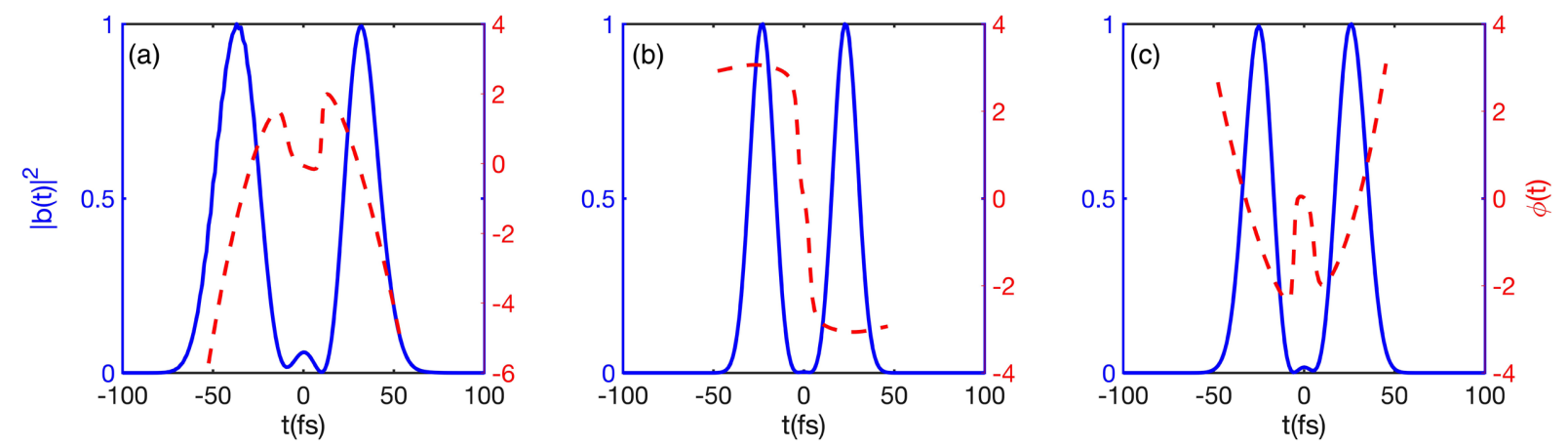

FIG. 4. Normalized square modulus (blue solid line, corresponding to the FEL pulse envelope) and phase (red dashed line, corresponding to the FEL phase) of the bunching factor in the time domain $b(t)$, obtained from the Fourier transform of the (calculated) bunching spectra indicated along the dashed lines in the insets of Fig. 3 for (a) GDD $=-4100 \mathrm{fs}^{2}$, (b) GDD $=0 \mathrm{fs}^{2}$, and (c) GDD $=2000 \mathrm{fs}^{2}$.

temporal domain, as shown in Fig. 4(a). Such pulses can be utilized in a pump-probe [32] or coherent control experiment $[11,23]$, with no need for an additional second seed pulse. The temporal separation between the pulses is controlled by the GDD (together with adjusting $A_{2}$ to maintain exact color separation) and is limited by the electron bunch length, while the color separation depends on the TL seed duration and is eventually limited by the FEL gain bandwidth.

The spectral map for zero GDD, Fig. 3(b), corresponds to a TL FEL pulse structure with a flat phase within the individual spikes, as demonstrated in Fig. 4(b), which shows the (calculated) temporal profile and phase for the case of the spectrum indicated by the vertical dashed line in the inset of Fig. 3(b). The relatively good agreement between experiment and theory confirms the discussion based on Fig. 2, from which we concluded that the FEL pulses are very close to the TL. This result demonstrates deterministic control of the FEL phase.

The case of a positive GDD of $2000 \mathrm{fs}^{2}$, Fig. 3(c), results in a similar spectral map as in Fig. 3(a), except that the sign of the chirp is reversed, as shown in Fig. 4(c). The small differences between Figs. 3(a) and 3(c) are due to different absolute values of GDD.

\section{CONCLUSION}

In summary, our results demonstrate that the EEHG scheme allows fine control over the spectrotemporal properties of FEL pulses in the soft-x-ray region. Starting from the optimized EEHG parameters, the seed laser bandwidth or chirp can be used to directly manipulate the spectral bandwidth of the FEL pulse, demonstrating deterministic control of the phase. Furthermore, adjusting the seed intensity, the FEL spectrum can be modified from a single- to a double-pulse structure with adjustable quadratic phase, as shown in Fig. 4. Increasing the seed energy above the values indicated by the dashed lines in the insets of Fig. 3, a more complicated pulse structure can be achieved (e.g., three pulses with a $\pi$ phase difference), which may be utilized in advanced spectroscopic techniques $[11,23]$. The ability to precisely control the spectrotemporal content of FEL pulses at these short wavelengths and the low sensitivity to electron-beam imperfections make EEHG the prime candidate for use in chirped-pulse amplification schemes [34].

\section{ACKNOWLEDGMENTS}

The authors acknowledge the continuous support of the FERMI team and the Elettra Sincrotrone Trieste operators during the experiment. E.H. was supported by the U.S. DOE BES Award no. 2017-SLAC-100382.

[1] W. S. Warren, H. Rabitz, and M. Dahleh, Coherent control of quantum dynamics: The dream is alive, Science 259, 1581 (1993).

[2] M. Shapiro and P. Brumer, Coherent control of molecular dynamics, Rep. Prog. Phys. 66, 859 (2003).

[3] Y. Silberberg, Quantum coherent control for nonlinear spectroscopy and microscopy, Annu. Rev. Phys. Chem. 60, 277 (2009).

[4] A. Assion, T. Baumert et al., Control of chemical reactions by feedback-optimized phase-shaped femtosecond laser pulses, Science 282, 919 (1998).

[5] P. Nuernberger, D. Wolpert, H. Weiss, and G. Gerber, Femtosecond quantum control of molecular bond formation, Proc. Natl. Acad. Sci. (U.S.A.) 107, 10366 (2010).

[6] H. J. Williams, L. Caldwell, N. J. Fitch, S. Truppe, J. Rodewald, E. A. Hinds, B. E. Sauer, and M. R. Tarbutt, Magnetic Trapping and Coherent Control of Laser-Cooled Molecules, Phys. Rev. Lett. 120, 163201 (2018).

[7] T. Bosma et al., Identification and tunable optical coherent control of transition-metal spins in silicon carbide, Quantum Information 4, 48 (2018).

[8] W. Ackermann et al., Operation of a free-electron laser from the extreme ultraviolet to the water window, Nat. Photonics 1, 336 (2007). 
[9] P. Emma et al., First lasing and operation of an ångstromwavelength free-electron laser, Nat. Photonics 4, 641 (2010).

[10] S. V. Milton et al., Exponential gain and saturation of a self-amplified spontaneous emission free-electron laser, Science 292, 2037 (2001).

[11] K. C. Prince et al., Coherent control with a shortwavelength free-electron laser, Nat. Photonics 10, 176 (2016).

[12] P. Kumar Maroju et al., Attosecond pulse shaping using a seeded free-electron laser, Nature (London) 578, 386 (2020).

[13] E. Allaria et al., Highly coherent and stable pulses from the FERMI seeded free-electron laser in the extreme ultraviolet, Nat. Photonics 6, 699 (2012).

[14] E. Allaria et al., Two-stage seeded soft-X-ray free-electron laser, Nat. Photonics 7, 913 (2013).

[15] E. L. Saldin, E. A. Schneidmiller, and M. V. Yurkov, Longitudinal space charge-driven microbunching instability in the TESLA Test Facility linac, Nucl. Instrum. Methods Phys. Res., Sect. A 528, 355 (2004).

[16] G. Stupakov, Using the Beam-Echo Effect for Generation of Short-Wavelength Radiation, Phys. Rev. Lett. 102, 074801 (2009).

[17] D. Xiang and G. Stupakov, Echo-enabled harmonic generation free electron laser, Phys. Rev. Accel. Beams 12, 030702 (2009).

[18] E. Hemsing, M. Dunning, C. Hast, T. O. Raubenheimer, S. Weathersby, and D. Xiang, Highly coherent vacuum ultraviolet radiation at the 15 th harmonic with echo-enabled harmonic generation technique, Phys. Rev. Accel. Beams 17, 070702 (2014).

[19] E. Hemsing, B. Garcia, Z. Huang, T. Raubenheimer, and D. Xiang, Sensitivity of echo enabled harmonic generation to sinusoidal electron beam energy structure, Phys. Rev. Accel. Beams 20, 060702 (2017).

[20] C. Feng, D. Haixiao et al., Coherent extreme ultraviolet free-electron laser with echo-enabled harmonic generation, Phys. Rev. Accel. Beams 22, 050703 (2019).

[21] E. Hemsing, M. Dunning, B. Garcia, C. Hast, T. Raubenheimer, G. Stupakov, and D. Xiang, Echo-enabled harmonics up to the 75th order from precisely tailored electron beams, Nat. Photonics 10, 512 (2016).

[22] P. Rebernik Ribič et al., Coherent soft X-ray pulses from an echo-enabled harmonic generation free-electron laser, Nat. Photonics 13, 555 (2019).

[23] A. Wituschek et al., Tracking attosecond electronic coherences using phase-manipulated extreme ultraviolet pulses, Nat. Commun. 11, 883 (2020).

[24] E. Hemsing, Minimum spectral bandwidth in echo seeded free electron lasers, Front. Physics 7, 35 (2019).

[25] D. Ratner, A. Fry, G. Stupakov, and W. White, Laser phase errors in seeded free electron lasers, Phys. Rev. Accel. Beams 15, 030702 (2012).

[26] The reader is also referred to SLAC Report No. SLACPUB-14639 (http://www.doi.org/10.2172/1029478) by G. Stupakov in which the problem of the seed laser chirp in HGHG and EEHG seeding was treated analytically for the first time.

[27] P. Finetti et al., Pulse Duration of Seeded Free-Electron Lasers, Phys. Rev. X 7, 021043 (2017).

[28] A. Borzsonyi, A. P. Kovacs, and K. Osvay, What we can learn about ultrashort pulses by linear optical methods, Appl. Sci. 3, 515 (2013).

[29] A. E. Siegman, Lasers (University Science Books, CA, 1986).

[30] G. De Ninno et al., Single-shot spectro-temporal characterization of XUV pulses from a seeded free-electron laser, Nat. Commun. 6, 8075 (2015).

[31] D. Gauthier et al., Spectrotemporal Shaping of Seeded Free-Electron Laser Pulses, Phys. Rev. Lett. 115, 114801 (2015).

[32] G. De Ninno, B. Mahieu, E. Allaria, L. Giannessi, and S. Spampinati, Chirped Seeded Free-Electron Lasers: Self-Standing Light Sources for Two-Color Pump-Probe Experiments, Phys. Rev. Lett. 110, 064801 (2013).

[33] D. Gauthier, B. Mahieu, and G. De Ninno, Direct spectrotemporal characterization of femtosecond extremeultraviolet pulses, Phys. Rev. A 88, 033849 (2013).

[34] D. Gauthier et al., Chirped pulse amplification in an extreme-ultraviolet free-electron laser, Nat. Commun. 7, 13688 (2016). 